\title{
Multiscale Capability in Rattlesnake using Contiguous Discontinuous Discretization of Self-Adjoint Angular Flux Equation
}

\author{
Weixiong Zheng ${ }^{1}$, Yaqi Wang ${ }^{2}$ and Mark D. DeHart ${ }^{3}$ \\ ${ }^{1}$ Department of Nuclear Engineering \\ Texas A\&M University \\ TAMU 3133 \\ College Station, TX 77843-3133 \\ ${ }^{2}$ Nuclear Engineering Methods Development Department \\ Idaho National Laboratory \\ P.O. Box 1625 \\ Idaho Falls, ID 83415-3840 \\ ${ }^{3}$ Reactor Physics Design and Analysis Department \\ Idaho National Laboratory \\ P.O. Box 1625 \\ Idaho Falls, ID 83415-3840
}

September 2016

INL is a U.S. Department of Energy National Laboratory operated by Battelle Energy Alliance 


\section{DISCLAIMER}

This information was prepared as an account of work sponsored by an agency of the U.S. Government. Neither the U.S. Government nor any agency thereof, nor any of their employees, makes any warranty, expressed or implied, or assumes any legal liability or responsibility for the accuracy, completeness, or usefulness, of any information, apparatus, product, or process disclosed, or represents that its use would not infringe privately owned rights. References herein to any specific commercial product, process, or service by trade name, trade mark, manufacturer, or otherwise, does not necessarily constitute or imply its endorsement, recommendation, or favoring by the U.S. Government or any agency thereof. The views and opinions of authors expressed herein do not necessarily state or reflect those of the U.S. Government or any agency thereof. 


\section{Multiscale Capability in Rattlesnake using Contiguous Discontinuous Discretization of Self-Adjoint Angular Flux Equation}

Weixiong Zheng, Yaqi Wang and Mark DeHart

September 2016

Idaho National Laboratory

Nuclear Systems Design and Analysis

Idaho Falls, Idaho 83415

http://www.inl.gov

Prepared for the

U.S. Department of Energy

Office of Nuclear Energy

Under DOE Idaho Operations Office

Contract DE-AC07-05ID14517 



\section{Contents}

1 Introduction and Motivation $\quad 1$

2 Angularly Continuous Weak Forms $\quad 2$

2.1 Another view of SAAF from a weighted least-squares functional . . . . . . . . . . . . . . . 2

2.2 Contiguous-discontinuous $(\mathrm{CD})$ weighted least-squares functional $\ldots \ldots \ldots \ldots$

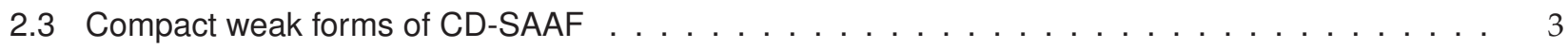

3 Angular Discretization $\quad 5$

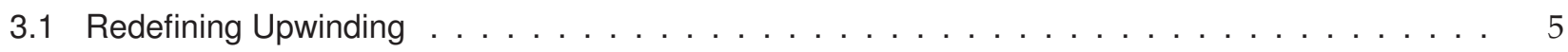

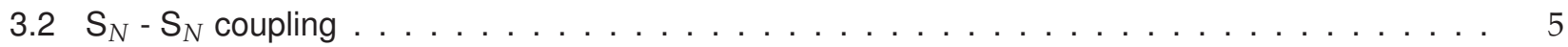

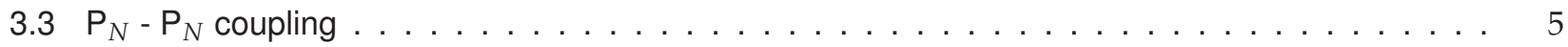

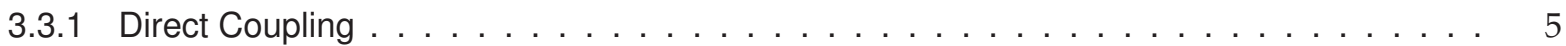

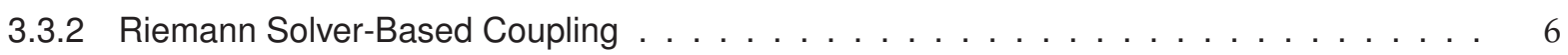

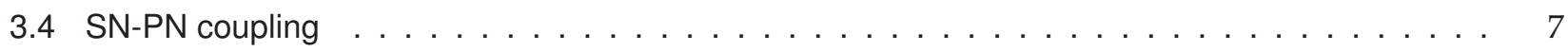

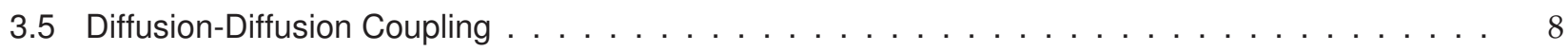

4 Numerical Tests $\quad 9$

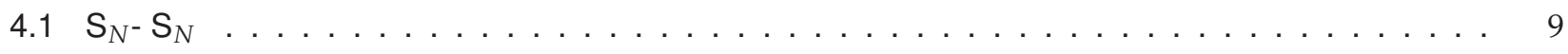

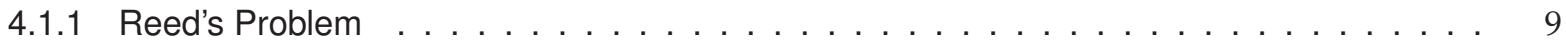

4.1 .2 Two-Region Test . . . . . . . . . . . . . . . . . . . 9

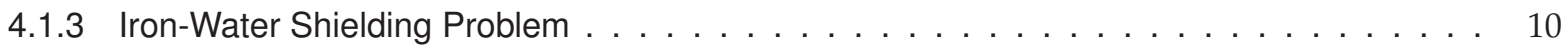

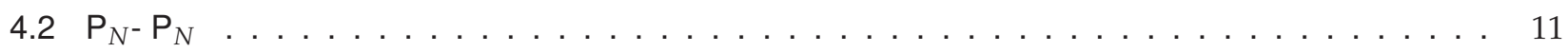

4.2 .1 Modified Reed's problem $\ldots \ldots \ldots \ldots \ldots \ldots \ldots$

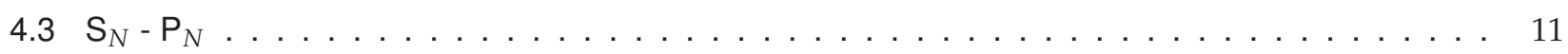

4.3 .1 Reed's problem . . . . . . . . . . . . . . . . . . . 11

4.3.2 Quasi-1D Modified Reed's Problem . . . . . . . . . . . . . . . . 11

4.3 .3 Poison Problem . . . . . . . . . . . . . . . . . . . . . . . . . . . 13

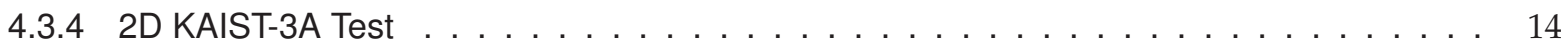

5 Conclusions and Future Work $\quad 16$ 


\section{List of Figures}

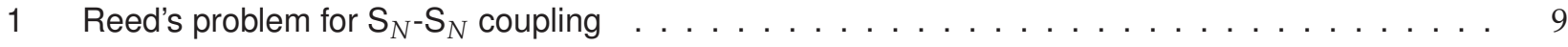

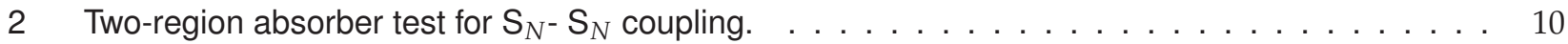

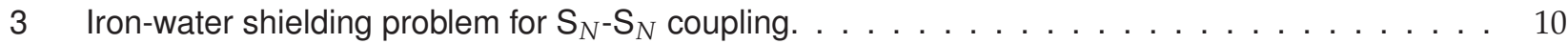

4 Modified Reed's problem with different order $P_{N}$ angular disretizations $\ldots \ldots \ldots \ldots$

$5 \quad$ Modified Reed's problem absorption rate errors for multiple schemes for $x \in(2,3) \mathrm{cm} \ldots \ldots$

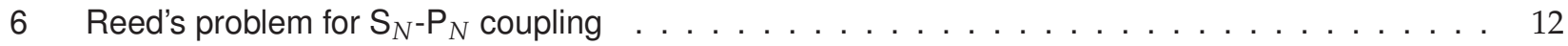

7 Quasi 1D modified Reed's problem for $\mathrm{S}_{N}-\mathrm{P}_{N}$ coupling compared with mortar implementation of

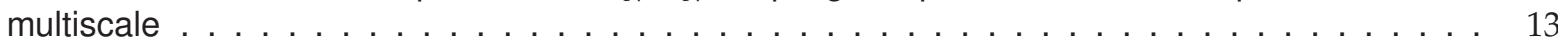

8 Line plots for quasi 1D modified Reed's problem. . . . . . . . . . . . . . . . . . . . . . 13

9 2D KAIST-3A problem configuration and settings for $S_{N}-P_{N}$ coupling subdomains . . . . . . . 14

10 The 7 -th group flux of KAIST-3A benchmark with CD-SAAF $\ldots \ldots \ldots \ldots$ 


\section{List of Tables}

$1 k_{\text {eff }}$ results for CFEM-SAAF-S ${ }_{16}$ with different layers of uniform refinements $\ldots \ldots \ldots 14$

$2 k_{\text {eff }}$ Results for SAAF-S 16 in Absorber Coupled with Different Angular Schemes in Fuel Meat, with 5 Layers of Uniform Refinement . . . . . . . . . . . . . . . . . . . . . . . . . . . 14 


\section{Introduction and Motivation}

Multiscale is a concept with which the entire spatial solution domain is divided into several subdomains where discretization schemes with different angular, space, energy resolutions can be applied [1, 2, 3, 4, 5, 6, 7]. The multiscale capability allows the transport solver to zoom into the domains which are of most interest without investing corresponding computing resources elsewhere. Consequently the computational efforts can be significantly reduced without significantly sacrificing the accuracy on the quantities of interest. Lower resolution in subdomains within a problem are typically made possible though spatial homogenization and energy condensation. General equivalencing needs be applied to minimize the errors caused by the homogenization and condensation [8]. The multiscale approach can also be used for problems in which general equivalence cannot be applied accurately for certain subdomains, for example, within TREAT simulations, the experiment region contained within full core.

A multiscale capability was first implemented within Rattlesnake using the mortar finite element method (MFEM) framework provided by MOOSE [7]. The interface condition for coupling $S_{N}$ (discrete ordinates method, suitable for heterogeneous subdomains) and $\mathrm{P}_{N}$ (spherical harmonics expansion method, which is better for subdomains with homogenization and condensation) with Lagrange multipliers defined on the subdomain interface or mortar interface were tested and proven to function correctly. However, the Lagrange multiplier-based approach requires certain constraints for domain partitioning (the domain cannot be partitioned along the interface) with $\mathrm{MPI}$, which is not currently supported by the framework. Hence, to more fully exploit the strength of parallel computing, we propose a new upwinding approach in this report. In this approach, the out-going angular fluxes of all the subdomains are the input of the neighboring subdomains. Because this does not require the variables to be defined on the interface, it can be implemented with the existing interface kernels provided by MOOSE without any constraints on the domain partitioning. However, it does come with the constraint that the mesh be conforming, i.e. there are no hanging nodes along the subdomain interface. This should not be a major issue, because users can (and generally do) generate a conforming mesh for their simulations. The upwinding approach can be implemented with the MFEM framework, which is necessary when the mesh is not conforming. This scheme also introduces the asymmetry of the streaming operator, which could make the problem harder to solve. It is noted that this is not necessary a drawback of the upwinding scheme but rather a cost of multiscale since the Lagrange multiplier approach also creates a saddle point problem, which requires special treatments on the numerical solver side. In the long term, the framework should support both Lagrange multiplier and upwinding approaches in a similar way for conforming meshes without requiring the constraints on domain partitioning.

In this report, both $S_{N}$ and $P_{N}$ discretization are based upon the SAAF (self-adjoint angular flux) [9, 10, 11, 12, 13] formulation of the transport equation although other formulations of the transport equation can be applied. The SAAF equation can be classified as a second order form of the transport equation. It introduces the proper stabilization for the continuous finite element method (CFEM) to perform spatial discretization for the hyperbolic transport equation. With SAAF, the symmetrization of the streaming operator breaks the causality of the particle transport process, i.e., particles transmit information not only from behind, as is supposed to occur, but also transmit information forward. This can cause issues in certain problems, for example, the thin-thick contact problem where the solution could be distorted by the thick materials. With the upwinding scheme on the thin-thick interface, the causality can be partially restored.

In Section 2, we first introduce the weak form of the upwinding interface condition without angular discretization. We then apply $S_{N}-S_{N} S_{N}-P_{N}$ and $P_{N}-P_{N}$ for the interface condition. In the case of $S_{N}-P_{N}$ we can see that from $P_{N}$ to $S_{N}$ the angular fluxes are sampled on the out-going discrete directions and used as the condition for the $S_{N}$ side. On the other side, we have the Marshak boundary condition for $P_{N}$ [14]. In the case of $\mathrm{P}_{N}-\mathrm{P}_{N}$ we obtain the Riemann upwind scheme. We then present numerical results with a few test problems in Section 4. We then state our conclusions and propose future work areas. 


\section{Angularly Continuous Weak Forms}

\subsection{Another view of SAAF from a weighted least-squares functional}

We have previously derived the SAAF weak form from the weighted residual equation. The SAAF weak form can be derived with the weighted least-squares functional. Although this is not necessary for proposing the weak form for the upwinding interface condition, it gives another view for SAAF and makes the derivation clearer.

For notation simplicity, we will perform our derivation with the one-speed steady-state transport equation with isotropic scattering and vacuum boundary conditions. Extending the methodology to multigroup, eigenvalue or transient problems with anisotropic scattering and various boundary conditions is straightforward. The onespeed steady state particle transport equation for a specific direction with scattering can be generically expressed as:

$$
\begin{gathered}
L \psi=q_{\mathrm{s}} \\
L \equiv \vec{\Omega} \cdot \nabla+\sigma_{\mathrm{t}} \quad \text { and } q_{\mathrm{s}} \equiv \mathbf{S} \psi+q=\sigma_{\mathrm{s}} \phi+q,
\end{gathered}
$$

where $\psi(\vec{r}, \vec{\Omega})$ is the angular flux, $\vec{r} \in \mathcal{D}, \mathcal{D}$ is the spatial solution domain, $\mathcal{S}$ is the unit sphere containing $4 \pi$ of solid angle, $\phi(\vec{r}) \equiv \int_{\mathcal{S}} d \Omega \psi$ is the scalar flux, $L$ is the transport operator, $\mathbf{S}$ is the scattering operator and $q$ is distributed volumetric source. The boundary condition is

$$
\psi\left(\vec{r}_{b}, \vec{\Omega}\right)=\psi^{\mathrm{inc}}\left(\vec{r}_{b}, \vec{\Omega}\right), \vec{r}_{b} \in \partial \mathcal{D}, \vec{\Omega} \cdot \vec{n}_{b}<0,
$$

where $\vec{n}_{b}$ is the outward unit vector at a point $\vec{r}_{b}$ on the boundary.

Solving the transport equation with the weighted least-squares finite element approach is equivalent to solving the following minimization problem: given a trial space $V_{h}$, find $\psi \in V_{h}$ such that the following functional is minimized:

$$
\Gamma=\oint_{\mathcal{S}} d \Omega \int_{\mathcal{D}} \frac{1}{\sigma_{\mathrm{t}}}\left(L \psi-q_{\mathrm{s}}\right)^{2}+\int_{\vec{n} \cdot \vec{\Omega}<0} d \Omega \int_{\partial \mathcal{D}} d s|\vec{n} \cdot \vec{\Omega}|\left(\psi-\psi^{\text {inc }}\right)^{2}
$$

$\vec{n}$ is the outward normal vector of domain $\mathcal{D}$ on the surface. We can translate the above problem by performing variation into the following weak formulation: find $\psi \in V_{h}$ such that

$$
\oint_{\mathcal{S}} d \Omega \int_{\mathcal{D}} d \vec{r}\left[L \psi^{*} \frac{1}{\sigma_{\mathrm{t}}}\left(L \psi-q_{\mathrm{s}}\right)\right]+\int_{\vec{n} \cdot \vec{\Omega}<0} d \Omega \oint_{\partial \mathcal{D}} d s|\vec{n} \cdot \vec{\Omega}| \psi^{*}\left(\psi-\psi^{\text {inc }}\right)=0 .
$$

Recognizing that

$$
\psi=\frac{-\vec{\Omega} \cdot \nabla \psi+q_{\mathrm{s}}}{\sigma_{\mathrm{t}}}
$$

and expanding the transport operator, we obtain the SAAF weak formulation after doing integration by parts:

$$
\begin{array}{r}
\oint_{\mathcal{S}} d \Omega \int_{\mathcal{D}} d \vec{r}\left(\vec{\Omega} \cdot \nabla \psi^{*} \frac{1}{\sigma_{\mathrm{t}}} \vec{\Omega} \cdot \nabla \psi+\sigma_{\mathrm{t}} \psi^{*} \psi-\psi^{*} q_{\mathrm{s}}-\vec{\Omega} \cdot \nabla \psi^{*} \frac{q_{\mathrm{s}}}{\sigma_{\mathrm{t}}}\right) \\
+\int_{\vec{n} \cdot \vec{\Omega}>0} d \Omega \oint_{\partial \mathcal{D}} d s|\vec{n} \cdot \vec{\Omega}| \psi^{*} \psi-\int_{\vec{n} \cdot \vec{\Omega}<0} d \Omega \oint_{\partial \mathcal{D}} d s|\vec{n} \cdot \vec{\Omega}| \psi^{*} \psi^{\text {inc }}=0 .
\end{array}
$$

\subsection{Contiguous-discontinuous (CD) weighted least-squares functional}

Note that the weak form is based on a continuous basis so continuity is enforced everywhere in the domain. However, there are certain situation, such as on a material interface, where allowing discontinuity might be beneficial. 
We define the functional as follows:

$$
\begin{aligned}
\Gamma_{\mathrm{CD}}= & \sum_{\mathcal{D}_{\mathrm{i}} \subset \mathcal{D}}\left[\oint_{\mathcal{S}} d \Omega \int_{\mathcal{D}_{\mathrm{i}}} d \vec{r} \frac{1}{\sigma_{\mathrm{t}}}\left(L \psi_{\mathrm{i}}-q_{\mathrm{si}}\right)^{2}+\int_{\vec{n} \cdot \vec{\Omega}<0} d \Omega \oint_{\partial \mathcal{D}_{i}}|\vec{n} \cdot \vec{\Omega}|\left(\psi_{i}-\psi^{\mathrm{inc}}\right)^{2}\right] \\
& +\sum_{\mathcal{D}_{\mathrm{i}} \subset \mathcal{D}} \sum_{\Gamma_{\mathrm{ij}}} \int_{\vec{n}_{i} \cdot \vec{\Omega}<0} d \Omega \oint_{\Gamma_{\mathrm{ij}}} d s\left|\vec{n}_{\mathrm{i}} \cdot \vec{\Omega}\right|\left(\psi_{\mathrm{i}}-\psi_{\mathrm{j}}\right)^{2}
\end{aligned}
$$

where $\Gamma_{\mathrm{ij}}$ is the interface between $\mathcal{D}_{\mathrm{i}}$ and contiguous subdomain $\mathcal{D}_{\mathrm{j}}$ and $\vec{n}_{\mathrm{i}}$ is the outward normal of subdomain $\mathcal{D}_{\mathrm{i}}$ on the interface. $\partial \mathcal{D}_{i}$ is the part of the boundary attached to the subdomain $\mathcal{D}_{i}$. This then leads to the following contiguous-discontinuous formulation:

$$
\begin{array}{r}
\quad \sum_{\mathcal{D}_{\mathrm{i}} \subset \mathcal{D}} \oint_{\mathcal{S}} d \Omega \int_{\mathcal{D}_{\mathrm{i}}} d \vec{r}\left(\vec{\Omega} \cdot \nabla \psi_{\mathrm{i}}^{*} \frac{1}{\sigma_{\mathrm{t}}} \vec{\Omega} \cdot \nabla \psi_{\mathrm{i}}+\sigma_{\mathrm{t}} \psi_{\mathrm{i}}^{*} \psi_{\mathrm{i}}-\psi_{\mathrm{i}}^{*} q_{\mathrm{si}}-\vec{\Omega} \cdot \nabla \psi_{\mathrm{i}}^{*} \frac{q_{\mathrm{si}}}{\sigma_{\mathrm{t}}}\right) \\
+\sum_{\mathcal{D}_{\mathrm{i}} \subset \mathcal{D}}\left[\int_{\vec{n} \cdot \vec{\Omega}>0} d \Omega \oint_{\partial \mathcal{D}} d s|\vec{n} \cdot \vec{\Omega}| \psi^{*} \psi-\int_{\vec{n} \cdot \vec{\Omega}<0} d \Omega \oint_{\partial \mathcal{D}} d s|\vec{n} \cdot \vec{\Omega}| \psi^{*} \psi^{\mathrm{inc}}\right] \\
+\sum_{\mathcal{D}_{\mathrm{i}} \subset \mathcal{D}} \sum_{\Gamma_{\mathrm{ij}}} \oint_{\Gamma_{\mathrm{ij}}} d s\left(\int_{\vec{n}_{i} \cdot \vec{\Omega}>0} d \Omega\left|\vec{n}_{\mathrm{i}} \cdot \vec{\Omega}\right| \psi_{\mathrm{i}}^{*} \psi_{\mathrm{i}}-\int_{\vec{n}_{i} \cdot \vec{\Omega}<0} d \Omega\left|\vec{n}_{\mathrm{i}} \cdot \vec{\Omega}\right| \psi_{\mathrm{i}}^{*} \psi_{\mathrm{j}}\right)=0 .
\end{array}
$$

Intuitively, the formulation above resembles CFEM-SAAF within the subdomains and on domain boundaries but differs from it on interfaces. It is noted that by the formulation above, when assembling the system, every subdomain interface needs to be visited twice. It can then be shown that the interface terms are identical to upwinding flux. We will name the resulting scheme as CD-SAAF in the remainer of this report.

\subsection{Compact weak forms of CD-SAAF}

To simplify notations, we define the following inner products with generic functions $a$ and $b$ :

$$
\begin{gathered}
(a, b)_{\mathcal{D}_{\mathrm{i}}} \equiv \int_{\mathcal{D}_{\mathrm{i}}} d \vec{r} \oint_{\mathcal{S}} d \Omega a b \\
<a, b>_{\Gamma} \equiv \oint_{\Gamma} d s \oint_{\mathcal{S}} d \Omega|\vec{n} \cdot \vec{\Omega}| a b, \\
<a, b>_{\Gamma}^{+} \equiv \oint_{\Gamma} d s \int_{\vec{n} \cdot \vec{\Omega}>0} d \Omega|\vec{n} \cdot \vec{\Omega}| a b, \\
<a, b>_{\Gamma}^{-} \equiv \oint_{\Gamma} d s \int_{\vec{n} \cdot \vec{\Omega}<0} d \Omega|\vec{n} \cdot \vec{\Omega}| a b,
\end{gathered}
$$

where $\Gamma$ is either an interior or a boundary face and $\vec{n}$ is the normal vector on the face. While $\vec{n}$ can be arbitrarily oriented in the interior, it must be outward on the boundary.

The weak form of CD-SAAF can be re-expressed as:

$$
\begin{aligned}
\left(\frac{1}{\sigma_{\mathrm{t}}} \vec{\Omega} \cdot \nabla \psi^{*}, \vec{\Omega} \cdot \nabla \psi\right)_{\mathcal{D}} & +\left(\psi^{*}, \sigma_{\mathrm{t}} \psi\right)_{\mathcal{D}}-\left(\psi^{*}, q_{\mathrm{s}}\right)_{\mathcal{D}}-\left(\frac{1}{\sigma_{\mathrm{t}}} \vec{\Omega} \cdot \nabla \psi^{*}, q_{\mathrm{s}}\right)_{\mathcal{D}} \\
& -\left\langle\left[\psi^{*}\right], \psi_{-}\right\rangle_{\Gamma}+\left\langle\psi^{*}, \psi\right\rangle_{\partial \mathcal{D}}^{+}-\left\langle\psi^{*}, \psi^{\mathrm{inc}}\right\rangle_{\partial \mathcal{D}}^{-}=0,
\end{aligned}
$$

where $\Gamma$ is the interface between any two contiguous subdomains. Here we drop the subdomain subscript of fluxes and merged the similar terms of all subdomains together. Note that the angular flux can be discontinuous across subdomain interfaces. The jump in the angular flux is defined as:

$$
\begin{gathered}
{\left[\psi^{*}\right]:=\psi_{+}^{*}-\psi_{-,}^{*}} \\
\psi_{ \pm}^{*}:=\lim _{s \rightarrow 0^{+}} \psi^{*}(\vec{r} \pm s \vec{\Omega}) .
\end{gathered}
$$


More specifically, the weak form can be separated to the bilinear form $b_{\mathrm{CD}-\mathrm{SAAF}}\left(\psi^{*}, \psi\right)$ and linear form $l_{\mathrm{CD}-\mathrm{SAAF}}\left(\psi^{*}\right)$ such that $b\left(\psi^{*}, \psi\right)=l\left(\psi^{*}\right)$ for any test function within the same function space, where the solution is sought:

$$
\begin{aligned}
& b_{\mathrm{CD}-\mathrm{SAAF}}\left(\psi^{*}, \psi\right)=\left(\frac{1}{\sigma_{\mathrm{t}}} \vec{\Omega} \cdot \nabla \psi^{*}, \vec{\Omega} \cdot \nabla \psi\right)_{\mathcal{D}}+\left(\psi^{*}, \sigma_{\mathrm{t}} \psi\right)_{\mathcal{D}}-\left(\psi^{*}, \mathbf{S} \psi\right)_{\mathcal{D}}-\left(\frac{1}{\sigma_{\mathrm{t}}} \vec{\Omega} \cdot \nabla \psi^{*}, \mathbf{S} \psi\right)_{\mathcal{D}} \\
&+\left\langle\psi^{*}, \psi\right\rangle_{\partial \mathcal{D}}^{+} \underline{-\left\langle\left[\psi^{*}\right], \psi_{-}\right\rangle_{\Gamma^{\prime}}} \\
& l_{\mathrm{CD}-\operatorname{SAAF}}\left(\psi^{*}\right)=\left(\psi^{*}, q\right)_{\mathcal{D}}+\left(\frac{1}{\sigma_{\mathrm{t}}} \vec{\Omega} \cdot \nabla \psi^{*}, q\right)_{\mathcal{D}}+\left\langle\psi^{*}, \psi^{\mathrm{inc}}\right\rangle_{\partial \mathcal{D}}^{-} .
\end{aligned}
$$

This form is almost identical to the original SAAF weak form except an extra interface upwinding term as underlined above. 


\section{Angular Discretization}

\subsection{Redefining Upwinding}

The classic upwinding definition in Eq. (11b) is with respect to specific direction, which introduces difficulties when performing angular discretization. Therefore, we redefine the upwinding in a manner that will be easier to implement. When treating the interface upwinding, we always specify master and slave subdomains. We assign master subdomains with a higher angular resolution than its neighboring or slave subdomains. For instance, we always set $S_{N}$ and $P_{N}$ subdomains to be master and slave, respectively when we have an $S_{N}-P_{N}$ interface because we typically use $S_{N}$ for a higher angular resolution. We choose the subdomains with higher $P_{N}$ order as the master when $\mathrm{P}_{N}-\mathrm{P}_{N}$ interface is considered. For $\mathrm{S}_{N}-\mathrm{S}_{N}$ interface, the master can be assigned to any subdomain because the angular quadrature must be identical due to lack of angular interpolation at present. We set the normal vector to be pointing from master subdomain to slave subdomain. We give the classic definitions based on the normal vector:

$$
\psi^{\mp}=\lim _{s \rightarrow 0^{+}} \psi(\vec{r} \mp s \vec{n}) \quad \text { and } \quad \llbracket \psi^{*} \rrbracket=\psi^{+*}-\psi^{-*} .
$$

That being said, $\psi_{-}$is always fixed to be the master angular flux. Consequently, the interface term can be re-expressed as:

$$
\begin{aligned}
I & =-\left\langle\left[\psi^{*}\right], \psi_{-}\right\rangle_{\Gamma}=-\oint_{\Gamma} d s\left(\int_{\vec{n} \cdot \vec{\Omega}>0} d \Omega|\vec{n} \cdot \vec{\Omega}| \llbracket \psi^{*} \rrbracket \psi^{-}-\int_{\vec{n} \cdot \vec{\Omega}<0} d \Omega|\vec{n} \cdot \vec{\Omega}| \llbracket \psi^{*} \rrbracket \psi^{+}\right) \\
& =-\left\langle\llbracket \psi^{*} \rrbracket, \psi^{-}\right\rangle_{\Gamma}^{+}+\left\langle\llbracket \psi^{*} \rrbracket, \psi^{+}\right\rangle_{\Gamma}^{-} .
\end{aligned}
$$

\section{$3.2 \mathrm{~S}_{N}-\mathrm{S}_{N}$ coupling}

We solve the equation in a given set of directions specified by an angular quadrature $\left\{\vec{\Omega}_{m}, w_{m}, m=1, \cdots, M\right\}$ with $S_{N}$. If the angular quadratures on two adjacent subdomains are the same, the discretized version of the upwinding interface condition can be simply expressed as:

$$
I=-\sum_{\vec{n} \cdot \vec{\Omega}_{\mathrm{m}}>0} w_{\mathrm{m}}\left(\left|\vec{n} \cdot \vec{\Omega}_{\mathrm{m}}\right| \llbracket \psi_{\mathrm{m}}^{*} \rrbracket, \psi_{\mathrm{m}}^{-}\right)_{\Gamma}+\sum_{\vec{n} \cdot \vec{\Omega}_{\mathrm{m}}<0} w_{\mathrm{m}}\left(\left|\vec{n} \cdot \vec{\Omega}_{\mathrm{m}}\right| \llbracket \psi_{\mathrm{m}}^{*} \rrbracket, \psi_{\mathrm{m}}^{+}\right)_{\Gamma}^{\prime}
$$

with the surface integral for generic functions $a$ and $b$ on arbitrary surface $\mathcal{F}$ being defined as:

$$
(a, b)_{\mathcal{F}}=\oint_{\mathcal{F}} d s a b .
$$

This condition is the traditional upwinding scheme for the discontinuous finite element method (DFEM) transport [15].

\section{3 $\quad \mathbf{P}_{N}-\mathbf{P}_{N}$ coupling}

\subsubsection{Direct Coupling}

We define the $\mathrm{P}_{N}$ expansion of the angular flux with real-valued spherical harmonics:

$$
\psi(\vec{\Omega})=\vec{R}^{\top}(\vec{\Omega}) \vec{\Phi},
$$

where $\vec{R}(\vec{\Omega})$ and $\vec{\Phi}$ are column vectors of spherical harmonics and moments, respectively. We also define the matrices:

$$
\mathbf{L}^{ \pm}=\int_{\vec{n} \cdot \vec{\Omega} \gtrless 0} d \Omega|\vec{n} \cdot \vec{\Omega}| \vec{R}(\vec{\Omega}) \vec{R}^{\top}(\vec{\Omega}) .
$$


It can be seen that $\mathbf{L}^{ \pm}$are symmetric and positive definite. Also,

$$
\mathbf{L}^{-}=\mathbf{C L}^{+} \mathbf{C}
$$

where $\mathbf{C}$ is a diagonal matrix, whose non-zero entries on diagonal is $\left\{(-1)^{l}, l=0, \cdots, N ; m=-l, \cdots, l\right\} \cdot \mathbf{C}^{-1}$ is equal to $\mathbf{C}$. Both $\mathbf{L}^{+}$and $\mathbf{L}^{-}$are positive definite and their eigenvalues are identical. Also the rotation with respect to $\vec{n}$ does not change the eigen structure of these two matrices.

If we plug Eq. (17) back to Eq. (14), we see:

$$
\begin{array}{r}
I=\left\langle\vec{\Phi}^{-*}, \mathbf{L}^{+} \vec{\Phi}^{-}\right\rangle+\left\langle\vec{\Phi}^{+*}, \mathbf{L}^{-} \vec{\Phi}^{+}\right\rangle-\left\langle\vec{\Phi}^{+*}, \mathbf{L}^{-} \vec{\Phi}^{-}\right\rangle-\left\langle\vec{\Phi}^{-*}, \mathbf{L}^{+} \vec{\Phi}^{+}\right\rangle \\
=\left\langle\llbracket \vec{\Phi}^{*} \rrbracket,\left(\mathbf{L}^{+}-\mathbf{L}^{-}\right)\{\vec{\Phi}\}+\frac{\mathbf{L}^{+}+\mathbf{L}^{-}}{2} \llbracket \vec{\Phi} \rrbracket\right\rangle
\end{array}
$$

where $\{\vec{\Phi}\}=\left(\vec{\Phi}^{+}+\vec{\Phi}^{-}\right) / 2$.

\subsubsection{Riemann Solver-Based Coupling}

It is clear that the formulation above similar to the upwinding in $\mathrm{P}_{N^{-}}$DFEM. What is different here is that $\mathrm{P}_{N^{-}}$ DFEM upwinding is based on the eigenstructure of streaming matrices while Eq. (20) is not. An alternative is then to perform eigenvalue decomposition and reformulate the upwinding in an eigenstructure based way. The cause of the difference is that the original formulation for upwinding is for the transport equation before it is discretized in angle while the eigenstructure based method is the upwinding after $P_{N}$ angular discretization has been introduced.

The interface weak form can be rewritten as:

$$
I=\int_{\vec{n} \cdot \vec{\Omega}>0} d \Omega|\vec{n} \cdot \vec{\Omega}|\left(\llbracket \psi^{*} \rrbracket, \widetilde{\psi}\right)-\int_{\vec{n} \cdot \vec{\Omega}<0} d \Omega|\vec{n} \cdot \vec{\Omega}|\left(\llbracket \psi^{*} \rrbracket, \widetilde{\psi}\right),
$$

where $\widetilde{\psi}$ is the "proper" angular flux that needs to be specified. Expand all the angular flux with $\mathrm{P}_{N}$ method, we have:

$$
I\left(\vec{\Phi}^{-*}, \vec{\Phi}^{+*}, \widetilde{\Phi}\right)=\left\langle\vec{\Phi}^{-*},\left(\mathbf{L}^{+}-\mathbf{L}^{-}\right) \widetilde{\Phi}\right\rangle_{\Gamma}-\left\langle\vec{\Phi}^{+*},\left(\mathbf{L}^{+}-\mathbf{L}^{-}\right) \widetilde{\Phi}\right\rangle_{\Gamma} .
$$

To know how to apply upwinding for $\mathbf{L}^{+}-\mathbf{L}^{-}$, we need to know the eigenstructure of the matrix. Since $\mathbf{L}^{+}-\mathbf{L}^{-}$ is symmetric, eigenvalue decomposition give us:

$$
\left(\mathbf{L}^{+}-\mathbf{L}^{-}\right)=\mathbf{U} \Lambda \mathbf{U}^{\top}
$$

where $\Lambda$ is the diagonal matrix whose diagonal elements are the eigenvalues of $\mathbf{L}^{+}-\mathbf{L}^{-}$. $\mathbf{U}$ is a matrix whose columns are the eigenvectors of $\mathbf{L}^{+}-\mathbf{L}^{-}$. Before proceeding, we separate $\Lambda$ to be $\Lambda^{+}$, which contains only the positive eigenvalues of $\Lambda$, and $\Lambda^{-}$, which in turn contains the absolute values of negative eigenvalues. Accordingly, we have:

$$
\Lambda=\Lambda^{+}-\Lambda^{-} \text {. }
$$

Then we can define two new matrices:

$$
\mathbf{M}^{ \pm}=\mathbf{U} \Lambda^{ \pm} \mathbf{U}^{T}
$$

It is noted that

$$
\mathbf{M}^{+}-\mathbf{M}^{-}=\mathbf{U} \Lambda^{+} \mathbf{U}^{\top}-\mathbf{U} \Lambda^{-} \mathbf{U}^{\top}=\mathbf{U}\left(\Lambda^{+}-\Lambda^{-}\right) \mathbf{U}^{\top}=\mathbf{U} \Lambda \mathbf{U}^{\top}=\mathbf{L}^{+}-\mathbf{L}^{-} .
$$

With the separation, the interface weak form can be transformed to:

$$
I=\left\langle\vec{\Phi}^{-*}, \mathbf{M}^{+} \vec{\Phi}^{-}\right\rangle_{\Gamma}-\left\langle\vec{\Phi}^{-*}, \mathbf{M}^{-} \vec{\Phi}^{+}\right\rangle_{\Gamma}-\left\langle\vec{\Phi}^{+*}, \mathbf{M}^{+} \vec{\Phi}^{-}\right\rangle_{\Gamma}+\left\langle\vec{\Phi}^{+*}, \mathbf{M}^{-} \vec{\Phi}^{+}\right\rangle_{\Gamma} .
$$


Similarly, the weak form can be re-expressed in a upwinding manner:

$$
I=\left\langle\llbracket \vec{\Phi}^{*} \rrbracket,\left(\mathbf{M}^{+}-\mathbf{M}^{-}\right)\{\vec{\Phi}\}+\frac{\mathbf{M}^{+}+\mathbf{M}^{-}}{2} \llbracket \vec{\Phi} \rrbracket\right\rangle
$$

therefore

$$
I=\left\langle\llbracket \vec{\Phi} * \rrbracket,\left(\mathbf{L}^{+}-\mathbf{L}^{-}\right)\{\vec{\Phi}\}+\frac{\mathbf{M}^{+}+\mathbf{M}^{-}}{2} \llbracket \vec{\Phi} \rrbracket\right\rangle .
$$

At the end, the new upwinding method differs from the original upwinding in the dissipation portion. Both $\mathrm{P}_{N}$ upwinding schemes Eq. (20) and Eq. (28) are implemented in Rattlesnake. The $\mathrm{P}_{N}$ orders on both sides are different, and the matrices are no longer square.

If we further proceed, we know:

$$
\mathbf{M}^{+}+\mathbf{M}^{-}=\mathbf{U} \Lambda^{+} \mathbf{U}^{\top}+\mathbf{U} \Lambda^{-} \mathbf{U}^{\top}=\mathbf{U}\left(\Lambda^{+}+\Lambda^{-}\right) \mathbf{U}^{\top}=\sum_{\mathrm{k}} \vec{r}_{\mathrm{k}}\left|\lambda_{\mathrm{k}}\right| \vec{r}_{\mathrm{k}}^{\top}
$$

where $\left(\lambda_{\mathrm{k}}, \vec{r}_{\mathrm{k}}\right)$ is the $k^{\text {th }}$ eigenpair of $\mathbf{L}^{+}-\mathbf{L}^{-}$. Introduce this back to (28), we will have the upwinding represented by the Roe type Riemann solver:

$$
I=\left\langle\llbracket \vec{\Phi}^{*} \rrbracket,\left(\mathbf{L}^{+}-\mathbf{L}^{-}\right)\{\vec{\Phi}\}+\frac{\sum_{\mathrm{k}} \vec{r}_{\mathrm{k}}\left|\lambda_{\mathrm{k}}\right| \vec{r}_{\mathrm{k}}^{\top}}{2} \llbracket \vec{\Phi} \rrbracket\right\rangle .
$$

\subsection{SN-PN coupling}

We always choose $S_{N}$ to be the master subdomain such that the normal vector $\vec{n}$ is always fixed to be from $S_{N}$ to $P_{N}$. We separate the weak form into four different terms based on what the weight and basis functions are, i.e.:

$$
I=I_{\mathrm{S}_{N}-\mathrm{S}_{N}}+I_{\mathrm{S}_{N}-\mathrm{P}_{N}}+I_{\mathrm{P}_{N}-\mathrm{S}_{N}}+I_{\mathrm{P}_{N}-\mathrm{P}_{N}}
$$

By performing all the angular integration with the quadrature identical to the $S_{N}$ subdomain, we can specifically write all four terms as:

$$
\begin{gathered}
I_{\mathrm{S}_{N}-\mathrm{S}_{N}}=\sum_{\vec{\Omega}_{m} \cdot \vec{n}_{m}>0}\left(\left|\vec{n} \cdot \vec{\Omega}_{\mathrm{m}}\right| \psi_{m}^{-*}, \psi_{m}^{-}\right), \\
I_{\mathrm{P}_{N}-\mathrm{S}_{N}}=-\sum_{\vec{\Omega}_{m} \cdot \vec{n}_{m}<0}\left(\left|\vec{n} \cdot \vec{\Omega}_{\mathrm{m}}\right| \psi_{m}^{-*}, \vec{R}\left(\vec{\Omega}_{m}\right) \vec{\Phi}^{+}\right), \\
I_{\mathrm{S}_{N}-\mathrm{P}_{N}}=-\left(\vec{\Phi}^{+*}, \vec{J}\right), \\
I_{\mathrm{P}_{N}-\mathrm{P}_{N}}=\left(\vec{\Phi}^{-*}, \overrightarrow{\mathbf{L}}^{-} \vec{\Phi}^{-}\right),
\end{gathered}
$$

where

$$
\begin{aligned}
\vec{J} & =\sum_{\vec{\Omega}_{m} \cdot \vec{n}_{m}>0}\left|\vec{n} \cdot \vec{\Omega}_{\mathrm{m}}\right| \vec{R}\left(\vec{\Omega}_{m}\right) \psi_{m}^{-} \\
\overline{\mathbf{L}}^{-} & =\sum_{\vec{\Omega}_{m} \cdot \vec{n}_{m}<0}\left|\vec{n} \cdot \vec{\Omega}_{\mathrm{m}}\right| \vec{R}^{T}\left(\vec{\Omega}_{m}\right) \vec{R}\left(\vec{\Omega}_{m}\right) .
\end{aligned}
$$

$\vec{J}$ are the partial outgoing flux moments with the first entry being the partial outgoing current and $\overline{\mathbf{L}}^{-}$is the discretized version of $\mathbf{L}^{-}$. 


\subsection{Diffusion-Diffusion Coupling}

The coupling between two diffusion schemes is to borrow the methodology developed for DFEM-diffusion that penalizes the jump of the scalar flux value. The difference from DFEM-diffusion is that in each subdomain CFEM is still applied to discretize the diffusion equation. We only apply the penalty method on the interface. We can arbitrarily choose one subdomain as the master and as always we fix the normal vector $\vec{n}$ pointing from master to slave. Further, every quantity belonging to a master/slave subdomain will be given a superscript " $\mp$ ". The definition of the penalty coefficient is given by [16]:

$$
\kappa=\max \left(p^{-}\left(p^{-}+1\right) \frac{D^{-}}{h^{-}}+p^{+}\left(p^{+}+1\right) \frac{D^{+}}{h^{+}}, \frac{1}{4}\right),
$$

where $p$ is the polynomial order; $D$ is diffusion coefficient and $h$ is the length of the cell orthogonal to interface. As an example of the 2D triangular mesh, $h=\frac{2 A}{L}$ where $A$ is the element area and $L$ is the element edge length on the interface. Thereafter, the interface bilinear form is formulated as:

$$
I=\left\langle\llbracket \Phi^{*} \rrbracket, \kappa \llbracket \Phi \rrbracket\right\rangle_{\Gamma}+\left\langle\left\{D \partial_{n} \Phi^{*}\right\}, \llbracket \Phi \rrbracket\right\rangle_{\Gamma}+\left\langle\llbracket \Phi^{*} \rrbracket,\left\{D \partial_{n} \Phi\right\}\right\rangle_{\Gamma},
$$

where $\Phi$ is the scalar flux and $\partial_{n} \Phi=\vec{n} \cdot \nabla \Phi$. 


\section{Numerical Tests}

Test results for $S_{N}-S_{N}, P_{N}-P_{N}$ and $S_{N}-P_{N}$ couplings will be presented. Reed-like problem [17] will be presented for all three schemes. The multi-region heterogeneous feature of the problem makes it ideal for testing purpose. For $S_{N}-P_{N}$ coupling, an eigenvalue problem named "poison problem" will be presented. Essentially, this problem is a 2D thick square of pure absorber surrounded by a thin fuel meat. Strong absorption makes it difficult for the CFEM-SAAF-S $S_{N}$ approach to get an accurate estimation of $k_{\text {eff }}$ even with many layers of spatial refinements. Finally, a more realistic problem, the KAIST-3A 2D test problem will be presented for an $S_{N}-P_{N}$ calculation. The results are organized into three subsections for $S_{N^{-}} S_{N}, P_{N^{-}} P_{N}$ and $S_{N^{-}} P_{N}$ respectively.

\section{$4.1 S_{N^{-}} S_{N}$}

\subsubsection{Reed's Problem}

The first test is for Reed's problem for $S_{N}-S_{N}$ coupling as illustrated in Figure 1. $S_{16}$ is used in the calculation. In the void, the Rattlesnake void treatment is utilized [13]. We can see that the flux profile is flat to a round-off level in the void region, which means that within void treatment, the subdomain-wise conservation is achieved. However, CFEM-SAAF-S ${ }_{N}$ with void treatment does not possess this property. In fact, with under-resolved mesh, CFEM-SAAF-S ${ }_{N}$ would present oscillations around the right interface in void as shown by the black dots in Figure 6. By partitioning subdomains with different materials, the subdomain-wise particle causality is fixed.

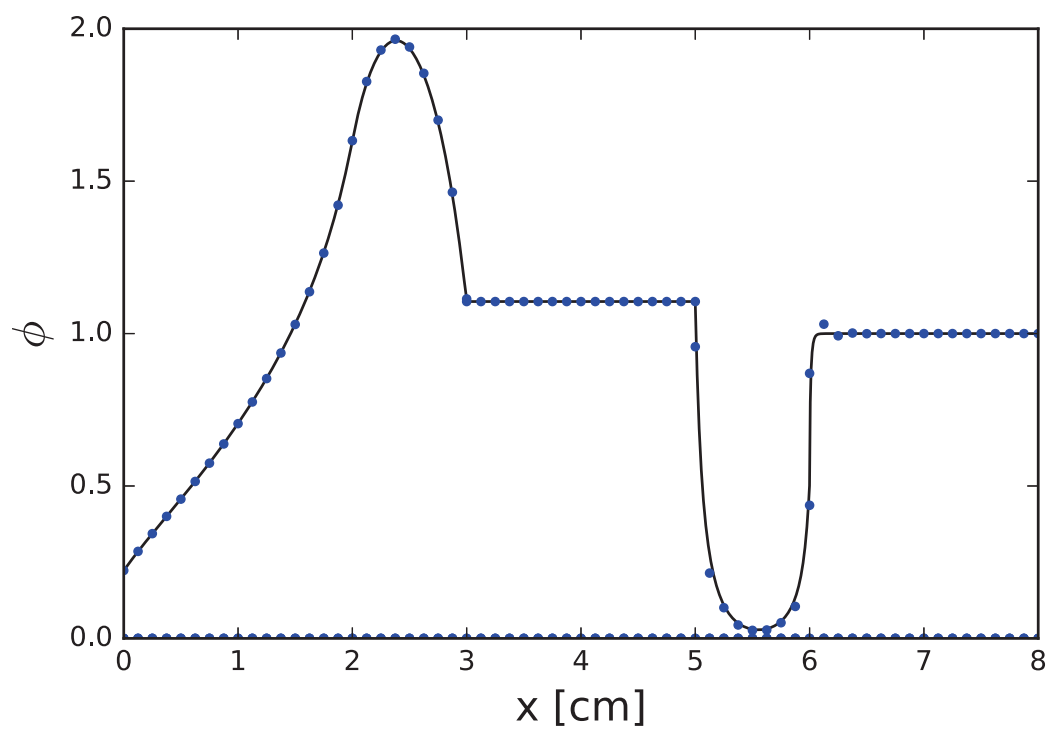

Figure 1 Reed's problem for $S_{N}-S_{N}$ coupling

\subsubsection{Two-Region Test}

The second test is a simple two-region absorber test. No source is presented in the test. An incident boundary condition is imposed on left side of the domain. Particles go through a thin absorber $\left(\sigma_{\mathrm{t}}=0.1, x \in(0,1) \mathrm{cm}\right)$ and then are attenuated by a thick absorber $\left(\sigma_{\mathrm{t}}=10, x \in(1,2) \mathrm{cm}\right)$ and exit at the right boundary. Figure $2 \mathrm{a}$ presents the comparison of the flux profiles with 16 cells for both CFEM-SAAF-S ${ }_{4}$ and CD-SAAF-S . With the additional degrees of freedom (DoFs) on the interface, the scalar flux in the thin material is leveled up accurately while the CFEM-SAAF's result is highly distorted in the thin material. Figure $2 b$ is the relative error of 
the leakage rate on the right side for different schemes. We also compared the least-squares (LS) $\mathrm{S}_{4}$ solution with the other two. Fixing up the causality not only brings accurate scalar flux profile in thin material, but also affects the accuracy of boundary leakage.

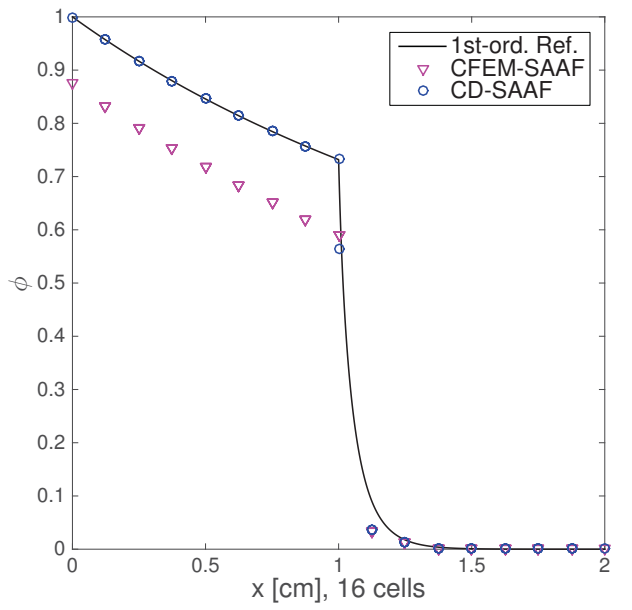

(a) Scalar flux profiles.

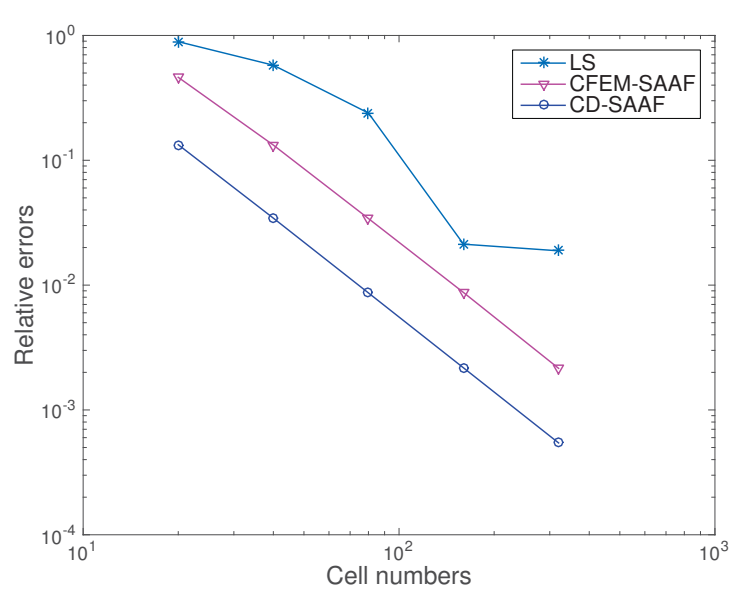

(b) Leakage rate errors on right side boundary.

Figure 2 Two-region absorber test for $S_{N^{-}} S_{N}$ coupling.

\subsubsection{Iron-Water Shielding Problem}

The iron-water problem is a $2 \mathrm{D}$ shielding problem with relatively thick materials. $\mathrm{S}_{2}$ is used in angle. A calculation with $1200 \times 1200$ cells is conducted to generate a reference solution. When measuring the absorption rate error in iron, we found both methods have roughly second order spatial convergence rates (see Figure 3). Yet, CD-SAAF delivers lower error magnitudes for all cell sizes.

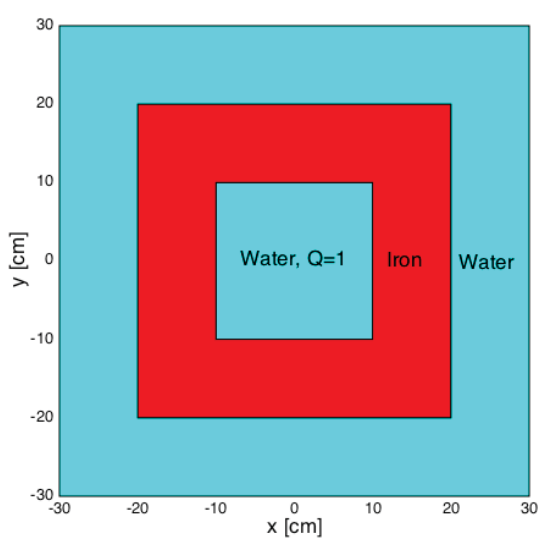

(a) Problem configuration.

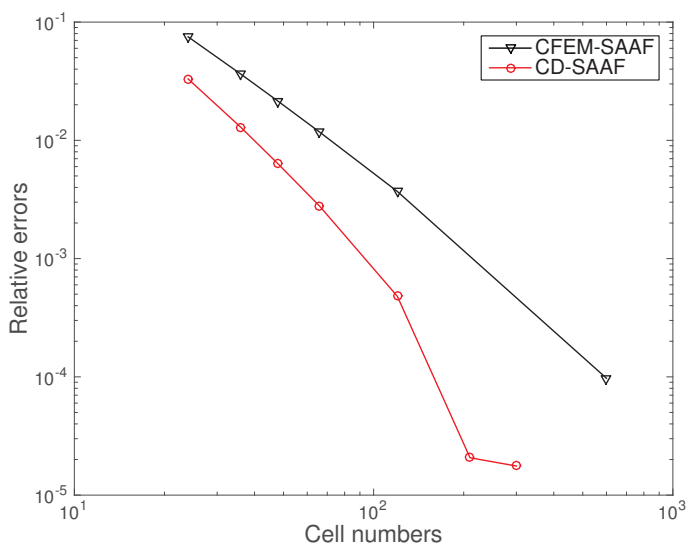

(b) Error of absorption rates in iron.

Figure 3 Iron-water shielding problem for $S_{N}-S_{N}$ coupling. 


\section{$4.2 \quad \mathbf{P}_{N^{-}} \mathbf{P}_{N}$}

\subsubsection{Modified Reed's problem}

The modified Reed's problem in the remainder of this report is based on the original Reed's problem but with the void region replaced with a thin absorber $\left(\sigma_{\mathrm{t}}=0.01\right)$. The mesh is refined such that the errors we see in Figure 4 are primarily from angular discretization. The $P_{1}-P_{1}$ curve presents discontinuous solution on the interfaces. This seems to be the results of the scheme trying to better fit the flux shape without increasing angular space in subdomains. Instead, the solution is detached on the interfaces. The Roe solver ("CD-SAAF- $\mathrm{P}_{N}$ : Riemann" shown in the legends) presents similar discontinuous solution on interfaces. However, increasing angular order from $P_{1}$ to $P_{5}$, we observe the discontinuity on the interfaces tend to vanish.

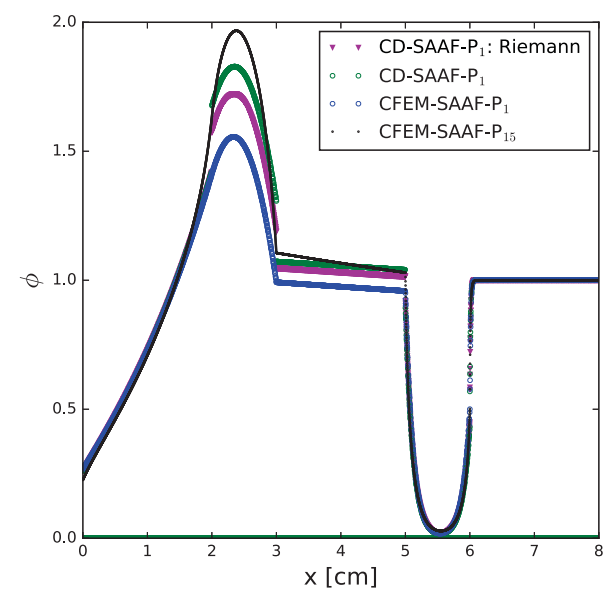

(a) CD-SAAF-P 1 with different upwindings.

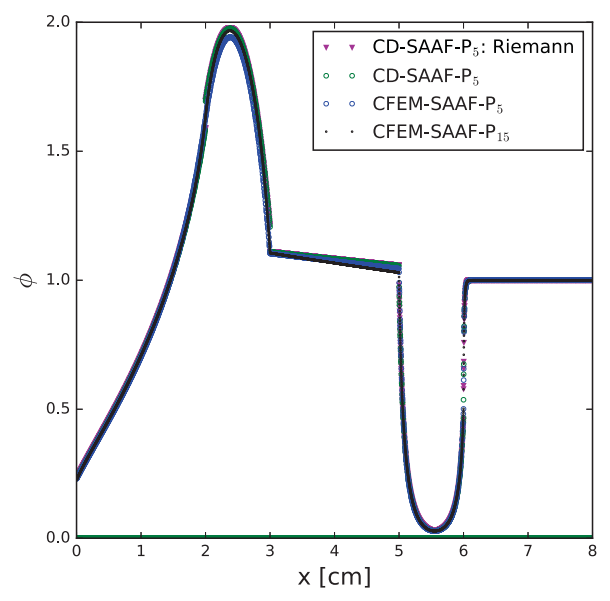

(b) CD-SAAF-P 5 with different upwindings.

Figure 4 Modified Reed's problem with different order $P_{N}$ angular disretizations

If we check the error of integral flux at the spots where schemes differ most (i.e. $x \in(2,3) \mathrm{cm}$ ), we see the error magnitudes are lowered significantly based on the reference of $S_{110}$ (see Figure 5). However, with increasing angular orders, all scheme errors decrease in the same direction.

\section{$4.3 \mathbf{S}_{N}-\mathbf{P}_{N}$}

\subsubsection{Reed's problem}

Reed's problem is the first test used as a demonstration of $S_{N}-P_{N}$ functionality. Green dots are for the hybrid calculation of $\mathrm{S}_{16}$ for $x \in(3,6) \mathrm{cm}$ and $\mathrm{P}_{5}$ elsewhere. The calculation presents acceptably accurate scalar flux profile in most regions. However, the scalar flux level in void is lower than the reference, which is from the CFEM-SAAF-S 16 . Apparently, high angular accuracy is required not only in void, but also in the subdomains contiguous to it in order to attain an accurate solution. Therefore, relatively high order $\mathrm{P}_{N}, \mathrm{P}_{13}$, is placed as a buffer layer for $x \in(2,3) \mathrm{cm}$ between $\mathrm{P}_{5}$ and $\mathrm{S}_{16}$. The purple triangles are for the new solution. As expected, the solution in void is more accurate by introducing the "buffer" region.

\subsubsection{Quasi-1D Modified Reed's Problem}

Higher dimensionality would introduce complexity and difficulty in certain situations. In $1 \mathrm{D}, \mathrm{S}_{N}$ and $\mathrm{P}_{N}$ are equivalent with certain types of $S_{N}$ quadrature. However, as spherical harmonics is the single set of angular 


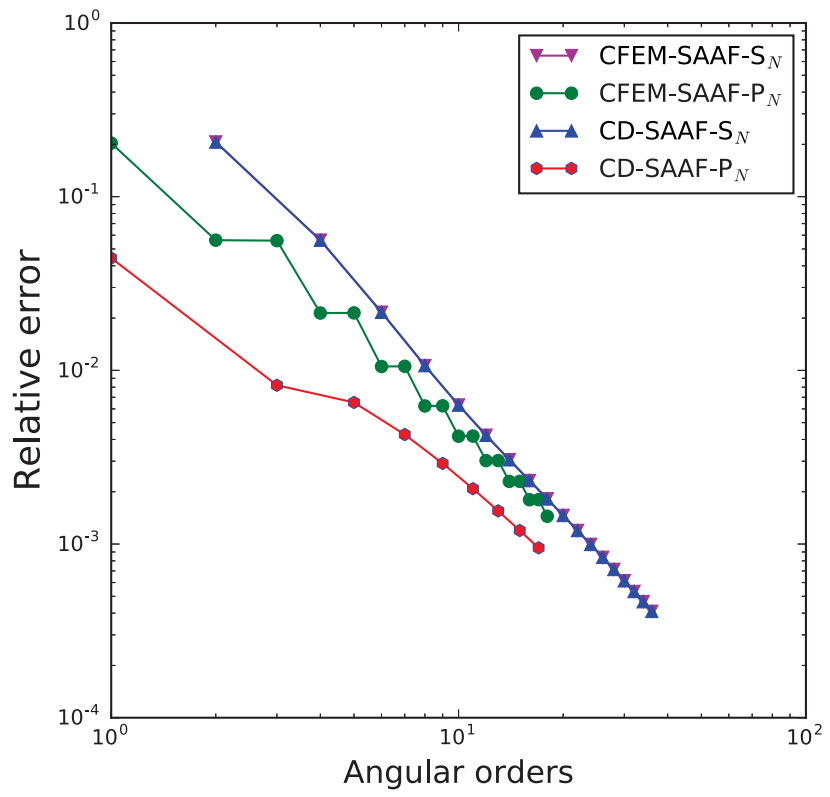

Figure 5 Modified Reed's problem absorption rate errors for multiple schemes for $x \in(2,3) \mathrm{cm}$.

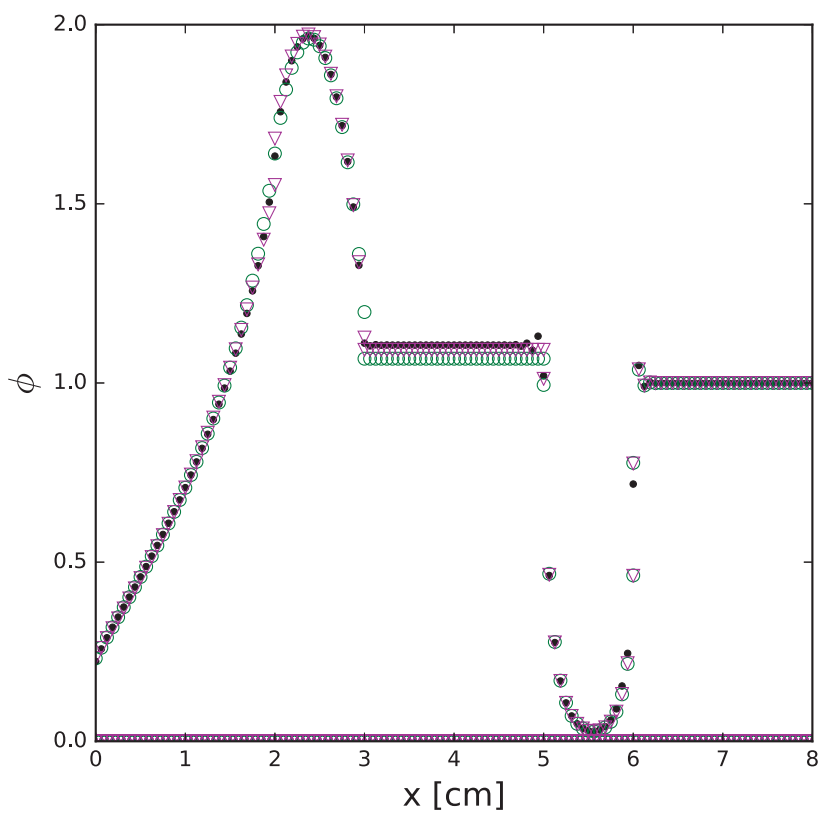

Figure 6 Reed's problem for $\mathrm{S}_{N}-\mathrm{P}_{N}$ coupling

bases which is rotationally invariant, $S_{N}$ with angular quadrature in multi-D it would not be equivalent to the $\mathrm{P}_{N}$ method. The discrepancy between these two methods causes oscillations around the coupling interface when coupling is realized by strongly enforcing angular flux continuity, as what a Lagrange multiplier interface condition would deliver[7]. A further check beyond the 1D test is thus necessary. We proposed to extend the modified Reed's problem to 2D. By making the upper and lower boundaries of a rectangular domain reflective, a $2 \mathrm{D}$ variation of modified Reed's problem is created. Along the x-axis, the setting is the same as for the 1D modified Reed's problem. Figure 7 presents the scalar flux for a hybrid scheme between $\mathrm{P}_{3}(x \in(3,5) \mathrm{cm})$ and $\mathrm{S}_{4}$ (elsewhere). Without angular smoothing around the coupling interfaces, necessary for Lagrange multiplier method, we still observe smooth solution in the domain. No obvious oscillation is seen around the interfaces with the upwinding scheme. 


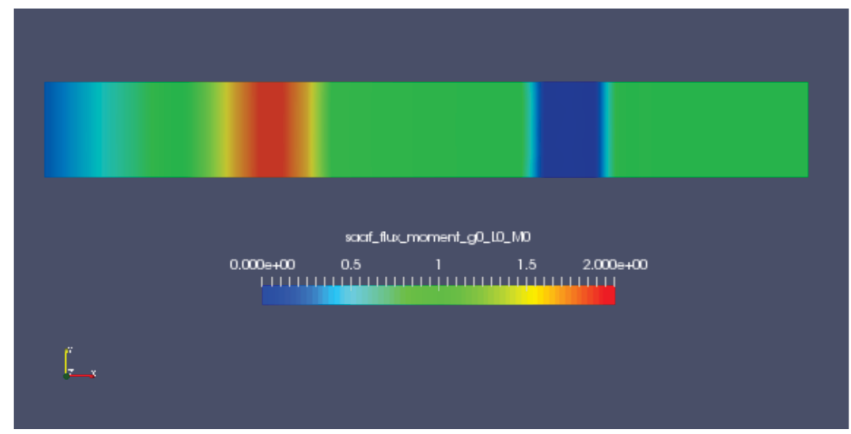

Figure 7 Quasi 1D modified Reed's problem for $S_{N}-P_{N}$ coupling compared with mortar implementation of multiscale

Figure $8 \mathrm{a}$ is a line plot along axis for $y=0.5 \mathrm{~cm}$. Lagrange multiplier and CD-SAAF solutions are produced using $4 \times 32$ cells. The reference is calculated with CFEM-SAAF-S ${ }_{4}$ with 2 additional levels of uniform refinement. CD-SAAF agrees with mortar solution in most regions but has much higher accuracy in the thin absorber, where the mortar solution is significantly distorted by neighboring thick materials. Lines along the y-axis near the $S_{N^{-}}$ $\mathrm{P}_{N}$ interfaces are shown in Figure 8b. Graphically, the scalar flux is flat and no oscillation is manifested around the interfaces.

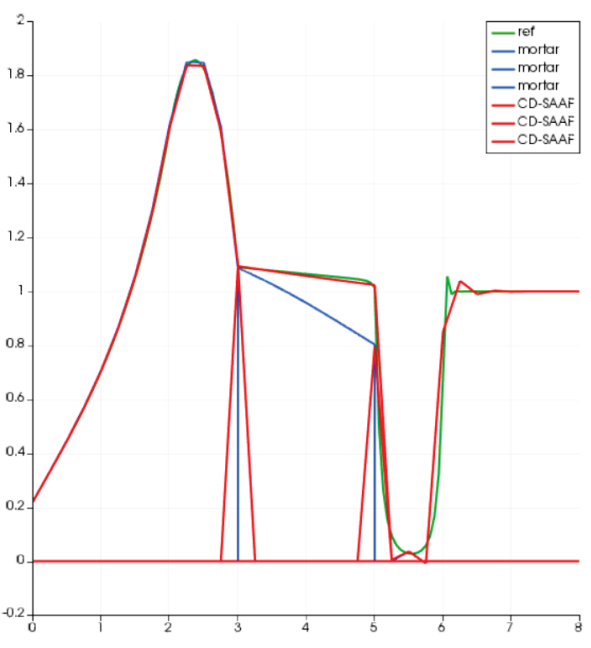

(a) Line plot for $y=0.5 \mathrm{~cm}$.

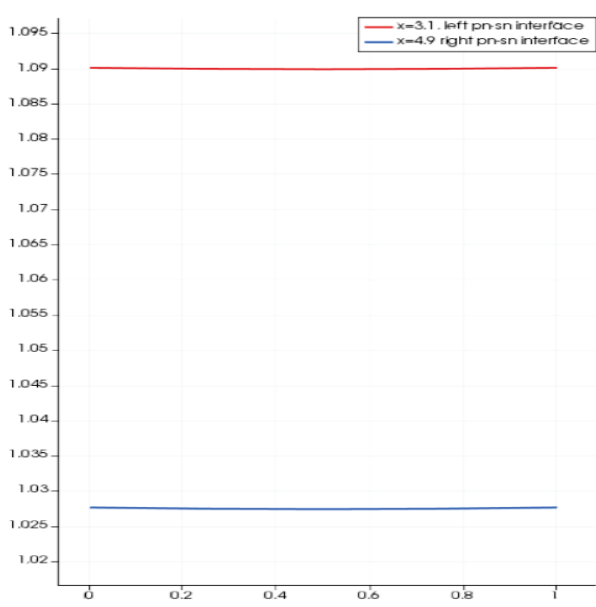

(b) Line plots along y direction around $\mathrm{S}_{N}-\mathrm{P}_{N}$ interfaces.

Figure 8 Line plots for quasi 1D modified Reed's problem.

\subsubsection{Poison Problem}

The "poison" problem is an eigenvalue problem characterized by a strong absorber in the middle of a 2D domain and surrounded by thin fuel meat. With the presence of the strong absorber, CFEM-SAAF-S ${ }_{16}$ needs a lot of refinement to get a converged $k_{\text {eff }}$. With 9 layers of uniform refinement ${ }^{1}$, CFEM-SAAF- $S_{16}$ gives a $k_{\text {eff }}$ of 0.89880 . We see slow convergence with increasing refinement from Table 1 . However, when setting an interface between the two materials, $S_{16}-S_{16}$ gives 0.89878 (see Table 2) with only five layers of uniform refinement. More importantly, when using $\mathrm{P}_{N}$ schemes in fuel instead, we gain comparable $k_{\text {eff }}$ value with about $P_{2}$ in the fuel, which is a significant savings in terms of total DoF.

\footnotetext{
${ }^{1}$ The total DoFs is over 150 million with $S_{16}$.
} 
Table $1 k_{\text {eff }}$ results for CFEM-SAAF-S 16 with different layers of uniform refinements

\begin{tabular}{|c|c|c|c|c|c|}
\hline Refinement layers & 5 & 6 & 7 & 8 & 9 \\
\hline$k_{\text {eff }}$ & 0.89675 & 0.89723 & 0.89779 & 0.89856 & 0.89880 \\
\hline
\end{tabular}

Table $2 k_{\text {eff }}$ Results for SAAF-S 16 in Absorber Coupled with Different Angular Schemes in Fuel Meat, with 5 Layers of Uniform Refinement

\begin{tabular}{|c|c|c|c|c|c|}
\hline Coupled schemes & $\mathrm{S}_{16}$ & $\mathrm{P}_{1}$ & $\mathrm{P}_{2}$ & $\mathrm{P}_{3}$ & $\mathrm{P}_{4}$ \\
\hline$k_{\text {eff }}$ & 0.89878 & 0.89852 & 0.89882 & 0.89879 & 0.89879 \\
\hline Coupled schemes & $\mathrm{P}_{5}$ & $\mathrm{P}_{6}$ & $\mathrm{P}_{7}$ & $\mathrm{P}_{8}$ & $\mathrm{P}_{9}$ \\
\hline$k_{\text {eff }}$ & 0.89879 & 0.89879 & 0.89879 & 0.89879 & 0.89879 \\
\hline
\end{tabular}

\subsubsection{D KAIST-3A Test}

The 2D 7 group KAIST-3A problem [18] is used for a more "realistic" test. The configuration and subdomain settings are shown in Figure 9.

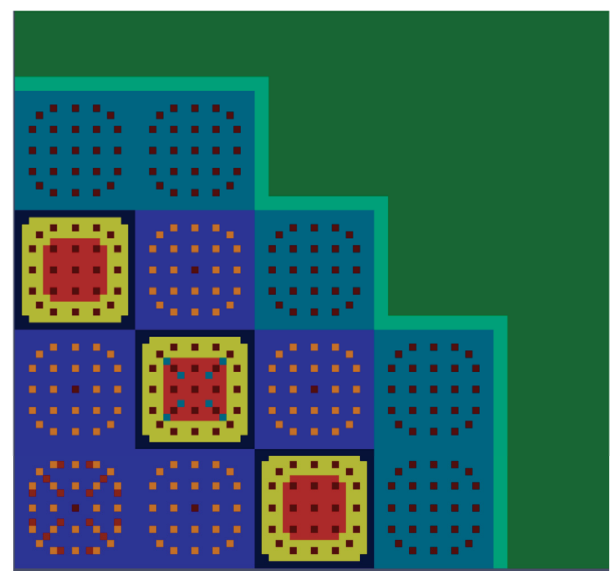

(a) Configuration of 2D KAIST-3A problem.

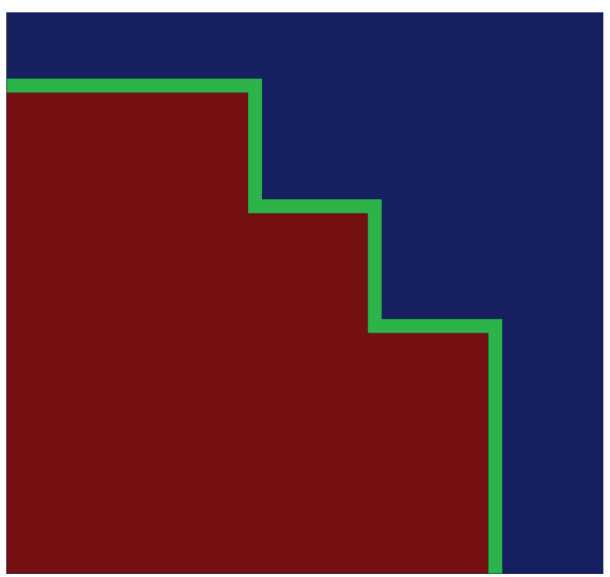

(b) Subdomain settings for $\mathrm{S}_{N}-\mathrm{P}_{N}$ coupling.

Figure 9 2D KAIST-3A problem configuration and settings for $S_{N}-P_{N}$ coupling subdomains

The 7-th group flux is plotted in Figure 10. We can see that the interface condition works properly with this relatively complicated geometry. 


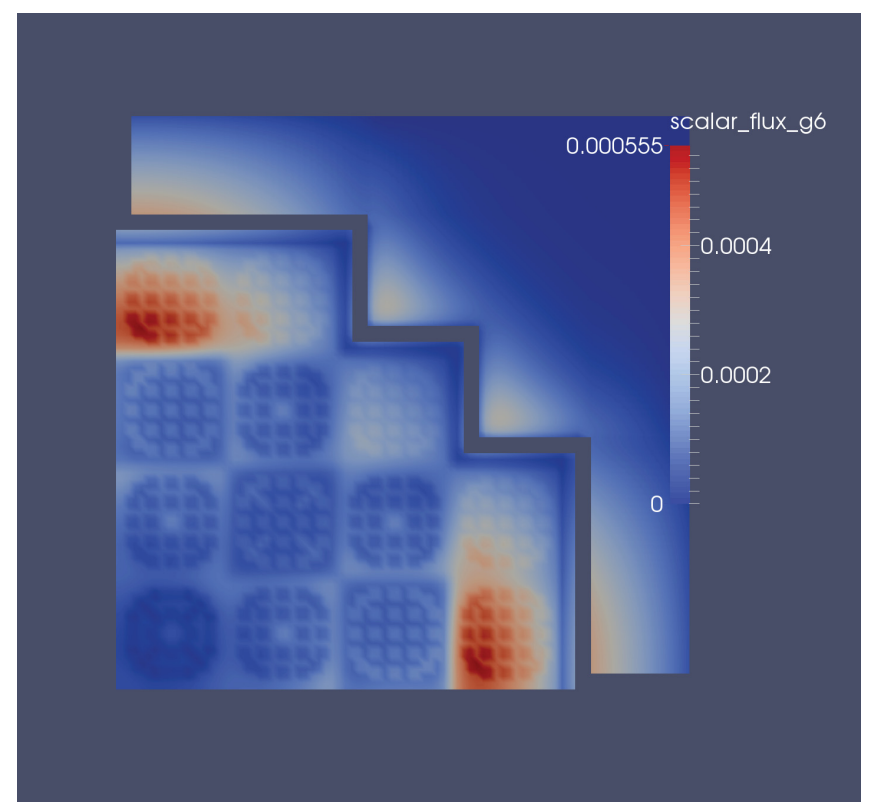

Figure 10 The 7-th group flux of KAIST-3A benchmark with CD-SAAF 


\section{Conclusions and Future Work}

In this report we present multiscale capabilities within Rattlesnake using an upwinding scheme. Although this multiscale approach requires the mesh to be conforming, it does not require constraints on the mesh partitioning. Hybrid $S_{N}-P_{N}$ interface conditions with upwinding are implemented and tested with both source and eigenvalue multi-dimensional problems. A Roe type Riemann solver is proposed for coupling $P_{N}$ to $P_{N}$ on the subdomain interface. The implementation allows flexible assignment of discretization schemes on the subdomains. Interface conditions are automatically added by Rattlesnake making the user interface fairly friendly. Because the upwinding scheme restores the causality of particle streaming on the subdomain interfaces, we see significant improvement in accuracy for certain test problems including a thin-thick material interface problem. The results suggest that a buffer region between high resolution subdomain and the low resolution subdomain is desirable. In the future, we will test the implementation with more realistic problems, ultimately for TREAT experiment modeling. Homogenization equivalence techniques should be incorporated into the multiscale simulation to reduce the errors introduced by spatial homogenization. Regions with different energy resolution also need to be considered.

\section{References}

[1] M. M. Nanneh and M. M. R. Williams. A diffusion-transport theory hybrid method for calculating neutron flux distributions in slab lattices. Atomkernenergie Kerntechnik, 47:221-224, 1985.

[2] Ricardo C. Barros, Musa Yavuz, Marcos P. de Abreu, Hermes Alves Filho, and José Antônio M. Mello. Progress in spectral nodal methods applied to discrete ordinates transport problems. Progress in Nuclear Energy, 33(1/2):117-154, 1998.

[3] Enrico Girardi and Jean-Michel Ruggieri. Mixed first- and second-order transport method using domain decomposition techniques for reactor core calculations. In Proc. International Conference on Supercomputing in Nuclear Application, Paris, France, 2003.

[4] Enrico Girardi, Jean-Michel Ruggieri, Patricia Sireta, and Guillaume Ritter. A new method for the treatment of local strong heterogeneities and its application to the phebus experimental facility. In PHYSOR 2004 The Physics of Fuel Cycles and Advanced Nuclear Systems : Global Developments, Chicago, Illinois, April 25-29 2004. American Nuclear Society, Lagrange Park, IL.

[5] Dmitriy Y. Anistratov and Nicholas D. Stehle. Computational transport methodology based on decomposition of a problem domain into transport and diffusive subdomains. Journal of Computational Physics, $231: 8009-8028,2012$.

[6] Sergiy Manolov, Jim E. Morel, and Cristian Rabiti. Hybrid Sn-diffusion and Sn-P3 transport calculations. In International Conference on Mathematics and Computational Methods Applied to Nuclear Science and Engineering, pages 2748-2758, Sun Valley, Idaho, May 5-9 2013.

[7] Yaqi Wang, Sebastian Schunert, Mark D. DeHart, and Richard C. Martineau. Hybrid PN-SN calculations with SAAF for the multiscale transport capability in Rattlesnake. In PHYSOR 2016 - Unifying Theory and Experiments in the 21st Century, Sun Valley, Idaho, USA, May 1-5, 20162016.

[8] K. S. Smith. Assembly homogenization techniques for light water reactor analysis. Progress in Nuclear Energy, 17,3:303-335, July 1986.

[9] J. E. Morel and J. M. McGhee. A self-adjoint angular flux equation. Nuclear Science and Engineering, 132:312-325, 1999.

[10] Jim E. Morel, B. Todd Adams, Taewan Noh, John M. McGhee, Thomas M. Evans, and Todd J. Urbatsch. Spatial discretizations for self-adjoint forms of the radiative transfer equations. Journal of Computational Physics, 214:12-40, 2006. 
[11] Jennifer Liscum-Powell. Finite element numerical solution of a self-adjoint transport equation for coupled electron-photon problems. Technical Report SAND2000-2017, Sandia National Laboratory, 2000.

[12] Jennifer L. Liscum-Powell, Anil K. Prinja, Jim E. Morel, and Leonard J. Lorence, Jr. Finite element solution of the self-adjoint angular flux equation for coupled electron-photon transport. Nuclear Science and Engineering, 142:270-291, 2002.

[13] Yaqi Wang, Hongbin Zhang, and Richard Martineau. Diffusion acceleration schemes for the self-adjoint angular flux formulation with a void treatment. Nuclear Science and Engineering, 176:201-225, 2014.

[14] E. E. Lewis and W. F. Miller. Computational Methods of Neutron Transport. John Wiley \& Sons, 1984.

[15] W. H. Reed and T. R. Hill. Triangular mesh methods for the neutron transport equation, Los Alamos Scientific Laboratory Report. Technical Report LA-UR-73-479, Los Alamos National Laboratory, 1973.

[16] Yaqi Wang. Adaptive Mesh Refinement Solution Techniques for the Multigroup SN Transport Equation Using a High-Order Discontinuous Finite Element Method. PhD thesis, Texas A\&M University, 2009.

[17] W. H. Reed. New difference schemes for the neutron transport equation. Nuclear Science and Engineering, 46(2):309-314, 1971.

[18] Nam Zin Cho. Benchmark problem 3A: MOX fuel-loaded small PWR core (MOX fuel with zoning) (7 group homogenized cells). Accessed on http://nurapt.kaist.ac.kr/benchmark/kaist_ben3a.pdf. 